\title{
Drying paraffin sections on hotplate unadvisable
}

Yang Guo, Yu Xiang and Zheng-Wei Yang*

*Correspondence: zwyang@nsmc.edu.cn

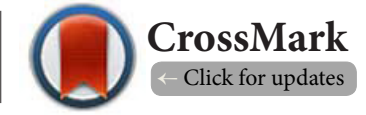

Morphometric Research Laboratory, North Sichuan Medical College, 234 Fujiang Road, Nanchong, Sichuan 637000, China.

\begin{abstract}
Previous study indicated that heating of paraffin sections at $90-140^{\circ} \mathrm{C}$ prevented their detachment from slides, but their thickness was reduced by half and staining affected with structures even disappearing. This study aimed to determine the effects of section drying at a temperature lower than the melting point of paraffin. 6 serial sections (thickness set at $10 \mu \mathrm{m}$ ) were cut from each of 12 paraffin embedded testicular tissue blocks, with 3 sections (control, randomly sampled from each block) placed vertically at room temperature immediately after floating onto slides from water bath $\left(37^{\circ} \mathrm{C}\right)$ and the other 3 heated on hotplate at $54^{\circ} \mathrm{C}$ for 2 hours for a complete drying. No sections detached during staining with hematoxylin. In comparison with control sections, the mean area of heated sections was unchanged but histological damage occurred. The damage varied from slight enlargement of the inter-tubular space or the tubular lumen (seen on most of the sections) to overturning or missing of the seminiferous tubules (seen on approximately 1/3 of the sections). Besides, the mean thickness of heated sections was reduced to $85 \%$ of control. We therefore concluded, among others, that it is not advisable to dry paraffin sections on hotplate.
\end{abstract}

Keywords: Histological techniques, paraffin sections, section attachment, section drying

\section{Introduction}

We recently found that heating of glycol methacrylateembedded sections on hotplate at $115-140^{\circ} \mathrm{C}$ before staining prevented detachment of the sections from microscope slides during staining [1]. And we further demonstrated that heating of the more commonly used paraffin-embedded sections at $90-140^{\circ} \mathrm{C}$ also prevented their detachment, but their thickness was reduced by half and staining affected with structures even disappearing [2]. This prompted us to ask whether section heating at a lower temperature would not only enhance section adhesion but also maintain section quality. Although it is often held that sections should be dried before staining [3,4], there is a paucity of literature regarding section drying as a means for the prevention of section loss $[1,2,5]$. In particular, few studies showed the effect of section drying on the flattening or area of paraffin sections that might be compressed while sectioning, and this would directly affect morphometric results such as nuclear numerical density [6]. This study was therefore carried out to determine the effects of paraffin section heating, at temperatures below the melting point of paraffin, on the section detachment, quality, thickness and area. We concluded unexpectedly that it is not advisable to dry paraffin sections on hotplate.

\section{Materials and methods}

12 testicular tissue blocks from normal adult rabbits, previously embedded in paraffin wax (JUNG-Histowax by Cambridge Instruments $\mathrm{GmbH}$, Nussloch, Germany; melting point $56^{\circ} \mathrm{C}$ ) in our laboratory 10 years ago, were utilized in the present study. Each block (semicircular) was half a testicular slice (2-3 $\mathrm{mm}$ in thickness) perpendicular to the long axis of the testis.

3 sets of serial sections were cut from each block at $10 \mu \mathrm{m}$ with a microtome (RM2235, Leica Biosystems Nussloch GmbH, Germany) and then floated onto adhesive glass slides (with positively charged surface, produced by Yifan Experiment, China) from distilled water bath $\left(37^{\circ} \mathrm{C}\right)$. Each set comprised 3 serial sections, which were randomly assigned to 3 groups designated as V-RT, $\mathrm{H}-37$ and $\mathrm{H}-54$, respectively. (To minimize thickness variation, a set of 4 serial sections were actually cut nonstop, with the first one being discarded).

In the group of V-RT, each section floated on slide was immediately placed in a slide box at room temperature at a vertical, upright position so that the water held under the section could drain readily down the slide. In the group of $\mathrm{H}-37$, section was first put horizontally on hotplate (HI1220, Leica Instruments $\mathrm{GmbH}$, Nussloch, Germany) at $37^{\circ} \mathrm{C}$ and then, approximately 20 minutes later when the peripheral 
wax of the section appeared dried, placed vertically in a slide box at room temperature. (The design considerations of the heating duration were: sections on hotplate at $37^{\circ} \mathrm{C}$ would not be dried up within a few hours; peripheral drying of the section implied an unlikely further flattening or stretching of the tissue section to a considerable extent.) In the group of $\mathrm{H}-54$, section was put on hotplate at $54^{\circ} \mathrm{C}$ for approximately 2 hours for a complete drying. (During this drying process, the wax did not melt, but some bobbles were seen formed under the section and broken before drying on most slides).

5-7 days post-sectioning, all sections were stained after a further overnight drying at $37^{\circ} \mathrm{C}$ in an incubator. The key staining procedures were dewaxing in xylene (for $3 \times 2$ minutes), staining in hematoxylin ( 1 minute), dehydration in $70 \%$ ethanol ( 1 minute) and absolute ethanol ( $1 \times 3$ minutes), clearing in xylene (1×2 minutes), and mounting with a neutral balsam.

Section was observed and measured on a computer screen equipped with a microscope (BX53, Olympus, Japan) and a stereology system (NewCAST, Visiopharm, Denmark). Using a $\times 1.25$ objective lens (PlanApo N, NA 0.04) for section observation, the area of each section was estimated by point counting [6], with an average of 30.4 test points being counted per section. Using a $\times 100$ oil immersion lens (UPlanSApo, NA 1.40) for section observation, the thickness of each section, randomly sampled from each set of 3 serial sections, was measured with a microcator, as we previously described $[1,2]$, at 12-17 fields sampled in a systematic random manner.

\section{Results}

No sections detached from slides during staining. Histologically, some damage occurred at approximately 1-12 places on each of the 30 sections from the $\mathrm{H}-54$ group while all the other 78 sections, including the other 6 from the $\mathrm{H}-54$ group, looked basically normal. The damage varied from slight enlargement of the inter-tubular space or the tubular lumen (seen on most of the sections) to overturning or missing of the tubules (seen on approximately $1 / 3$ of the sections), see Figure 1.

The mean section areas of 36 sets of serial sections were 31.4-55.9 $\mathrm{mm}^{2}$, without significant differences in the means among the 3 groups (one way repeated measures analysis of variance: $P=0.40$ ), see Table 1.

The mean thickness of sections in the group of V-RT slightly shrank by an average of $1.8 \%$ compared to the thickness 10 $\mu \mathrm{m}$ set by the microtome (Table 1). For comparison between the 3 groups, the mean section thickness in the group of $\mathrm{H}-54$, not $\mathrm{H} 37$, was consistently reduced to $84.9 \% \pm 4.4 \%(77.2 \%$ $90.3 \%$, ratio of the 2 mean thicknesses of the 2 sections from the same tissue block) of V-RT (Table 1). The mean coefficient of variation (SD divided by the mean of each section) of the 36 sections was $2.88 \% \pm 1.92 \%$.

\section{Discussion}

With random grouping of each set of 3 serial sections, which eliminated estimation bias resulting possibly from the presumable

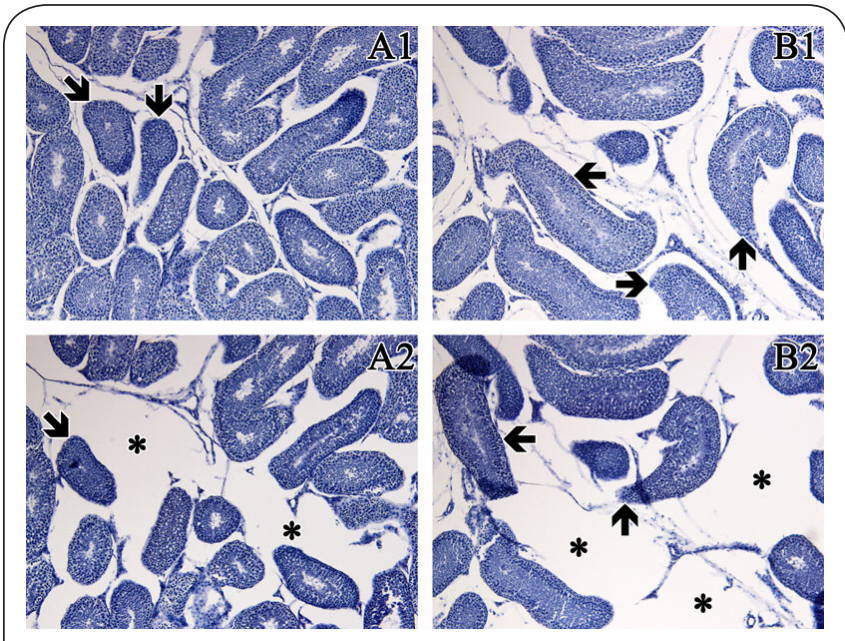

Figure 1. Micrographs (each with width $1.41 \mathrm{~mm}$ ) taken from adjacent serial paraffin-embedded testicular sections: the first (A1) and middle (A2) sections from a set of 3 serial sections, and the middle (B1) and last (B2) sections from another set of 3 serial sections. A1, section dried vertically at room temperature; $\mathbf{B} \mathbf{1}$, section dried horizontally on hotplate at $37^{\circ} \mathrm{C} ; \mathbf{A} 2$ and $\mathbf{B 2}$, sections dried horizontally on hotplate at $54^{\circ} \mathrm{C}$ (immediately after floating onto slide from water bath). Seminiferous tubule profiles indicated by $\boldsymbol{\downarrow}$ on $\mathbf{A} \mathbf{1}$ and $\boldsymbol{\leftarrow}$ and $\boldsymbol{\uparrow}$ on $\mathbf{B} \mathbf{1}$, overturned to the left on $\mathbf{A} \mathbf{2}$ and B2, respectively; tubules indicated by $\boldsymbol{\downarrow}$ on $\mathrm{A} 1$ and $\rightarrow$ on B1, missing on A2 and B2, respectively; ${ }^{*}$, abnormally large empty space between the tubules.

Table 1. Area and thickness of paraffin sections, $\bar{x} \pm \operatorname{SD}(n=12)$.

\begin{tabular}{llll}
\hline & V-RT & H-37 & H-54 \\
\hline Mean area of sections $\left(\mathrm{mm}^{2}\right)$ & $41.8 \pm 7.3$ & $42.3 \pm 7.0$ & $42.1 \pm 7.0$ \\
$\begin{array}{l}\text { Mean thickness of sections } \\
(\mu \mathrm{m})\end{array}$ & $9.82 \pm 0.32^{*}$ & $9.67 \pm 0.31^{*}$ & $8.34 \pm 0.49$ \\
\hline
\end{tabular}

V-RT: each section (thickness set at $10 \mu \mathrm{m}$ in cutting) floated on slide was immediately placed vertically at room temperature. H-37: section first put horizontally on hotplate at $37^{\circ} \mathrm{C}$ for 20 minutes and then placed vertically at room temperature. H-54: section put on hotplate at $54^{\circ} \mathrm{C}$ for 2 hours for a complete drying. ${ }^{\star} P \leq 0.05$ for comparison with the group of $\mathrm{H}-54$ (one way repeated measures analysis of variance in combination with the Student-NewmanKeuls method for multiple comparisons).

size difference between the sections, the present study provided evidence that drying paraffin sections on hotplate at $37^{\circ} \mathrm{C}$ or $54^{\circ} \mathrm{C}$ (below the melting point of paraffin) would not further flatten the sections or increase the section area. This suggested that sufficient flattening of the sections occurred in the water bath $\left(37^{\circ} \mathrm{C}\right)$ and/or the sections floated onto slides shortly dried or adhered to slides. This is a merit of paraffin sections in morphometric studies although it cannot correct their defects such as tissue shrinkage and section compression [6], see also below.

According to our trial, heating of paraffin sections (not dewaxed) on hotplate at $90^{\circ} \mathrm{C}$ or $140^{\circ} \mathrm{C}$ for 30 minutes would completely spoil the sections: almost no structures could be stained [2]. 
So we suppose that paraffin sections are better heated at temperatures below the melting point of paraffin, e.g., at $54^{\circ} \mathrm{C}$ (near the melting point $56^{\circ} \mathrm{C}$ ) as tried in this study. And it is recommended by technical books to heat paraffin sections at $45-50^{\circ} \mathrm{C}$ [3] or the melting point [4] for section attaching or stretching. To our surprise, this study revealed that heating of paraffin sections at $54^{\circ} \mathrm{C}$ damaged sections in terms of histology, and also induced a $15 \%$ reduction in the section thickness, which would potentially bias morphometric estimation. The histological damage must have been the result of impact (e.g., formation of vapor or bubbles) of water (under the section) during heating and the thickness reduction the result of too much drying.

Jones et al., (2001) reported that heating of paraffin sections at $80^{\circ} \mathrm{C}$ for 16 hours would only slightly decrease the intensity of staining, and suggested that paraffin sections be attached to slides overnight at $65^{\circ} \mathrm{C}$ for section attachment [5]. The heating was probably applied after dewaxing of the sections because otherwise the sections would be markedly damaged according to our experience (see above). We also noted that heating after dewaxing at $90^{\circ} \mathrm{C}$ for 30 minutes did not severely damage sections [2]. But such heating, at $90^{\circ} \mathrm{C}$ or $54^{\circ} \mathrm{C}$ as shown in our previous [2] and current studies, was compromised by the potential effect of marked section thickness reduction on morphometry.

Due to limitation of the section number and study design -the fact of no sections detaching from slides as a result, the present study failed to confirm the effect of section heating on the prevention of section detachment. However, our experience indicated the importance of the whole section attaching tightly to slide by draining the water under the section, and this was simply achieved by erecting the section immediately after mounting from water bath. Leaving a section flat on hotplate or table for long might increase the possibility of deforming (e.g., outspreading) the section by the water retained under the section and forming cavity between the slide and section when dried, thus resulting more likely in section detachment in staining.

Taken together, we consider it unadvisable to dry paraffin sections (freshly floated onto slides from water bath) on hotplate or at a horizontal position. When the drying temperature is high, the section will be destructed and compressed; when the temperature is low, there will be no drying effect or that the section maybe deformed. Putting sections upright is a simple and efficient way of section drying. It should be pointed out, however, that the conclusion is mainly based on study with a soft tissue (adult rabbit testis) embedded long ago. More studies are therefore needed for further confirmation although attachment of tissue sections to glass slides may not be affected considerably by types of tissue or storage of embedded tissue blocks.

\section{Competing interests}

The authors declare that they have no competing interests.

\section{Authors' contributions}

\begin{tabular}{|l|c|c|c|}
\hline Authors' contributions & YG & YX & ZWY \\
\hline Research concept and design & $\checkmark$ & -- & $\checkmark$ \\
\hline Collection and/or assembly of data & $\checkmark$ & $\checkmark$ & -- \\
\hline Data analysis and interpretation & -- & -- & $\checkmark$ \\
\hline Writing the article & -- & -- & $\checkmark$ \\
\hline Critical revision of the article & -- & -- & $\checkmark$ \\
\hline Final approval of article & $\checkmark$ & $\checkmark$ & $\checkmark$ \\
\hline Statistical analysis & -- & -- & $\checkmark$ \\
\hline
\end{tabular}

\section{Acknowledgement}

This study was supported by grants from the Research Development Program of North Sichuan Medical College (CBY13-A-QN35 \& CBY13-A-ZP05).

Publication history

EIC: Gaetano Giuseppe Magro, University of Catania, Italy. Received: 16-Sep-2016 Final Revised: 21-Oct-2016 Accepted: 30-Oct-2016 Published: 08-Nov-2016

\section{References}

1. Xiang $Y$ and Yang $Z W$. Detachment of methacrylate-embedded sections from microscope slides can be prevented by heating on hotplate. $J$ Histol Histopathol. 2014; 1:10. I Article

2. Xiang $Y$, Guo $Y$, Huang $X L$, Peng $B$ and Yang $Z W$. Effects of heating on the adhesion, thickness and staining of paraffin sections. Chinese Journal of Stereology and Image Analysis. 2015; 20:52-7.

3. Li JC and Li DM. Sectioning and staining techniques of tissue specimen. In Li JC (Ed.), Experimental Techniques of Histology and Embryology, 1st edition. Beijing: People's Medical Publishing House. 2010, 1-71.

4. Spencer LT and Bancroft JD. Microtomy: paraffin and frozen. In Suvarna SK, Layton C and Bancroft JD (Eds.), Bancroft's Theory and Practice of Histological Techniques, 7th edition. Churchill Livingstone, Elsevier. 2013, 125-38.

5. Jones WT, Stockard CR and Grizzle WE. Effects of time and temperature during attachment of sections to microscope slides on immunohistochemical detection of antigens. Biotech Histochem. 2001; 76:55-8. | Article | PubMed

6. Yang ZW. Essential Tools for Morphometric Studies of Biological Tissues: Practical Stereological Methods, 1st edition. Beijing: Science Press, 2012; 106-64.

\section{Citation:}

Guo Y, Xiang Y and Yang Z-W. Drying paraffin sections on hotplate unadvisable. J Histol Histopathol. 2016; 3:4. http://dx.doi.org/10.7243/2055-091X-3-4 